\title{
Multi-organ Segmentation Based on Spatially-Divided Probabilistic Atlas from 3D Abdominal CT Images
}

\author{
Chengwen $\mathrm{Chu}^{1}$, Masahiro Oda ${ }^{1}$, Takayuki Kitasaka ${ }^{2}$, Kazunari Misawa $^{3}$, \\ Michitaka Fujiwara $^{4}$, Yuichiro Hayashi ${ }^{5}$, Yukitaka Nimura ${ }^{5}$, \\ Daniel Rueckert ${ }^{6}$, and Kensaku Mori ${ }^{5}$ \\ 1 Graduate School of Information Science, Nagoya University, Nagoya, Japan \\ 2 Aichi Institute of Technology, Toyota, Japan \\ 3 Aichi Cancer Center Hospital, Nagoya, Japan \\ 4 Graduate School of Medicine, Nagoya University, Nagoya, Japan \\ ${ }^{5}$ Information and Communications Headquarters, Nagoya University, Japan \\ ${ }^{6}$ Imperial College London, London, UK
}

\begin{abstract}
This paper presents an automated multi-organ segmentation method for 3D abdominal CT images based on a spatially-divided probabilistic atlases. Most previous abdominal organ segmentation methods are ineffective to deal with the large differences among patients in organ shape and position in local areas. In this paper, we propose an automated multi-organ segmentation method based on a spatiallydivided probabilistic atlas, and solve this problem by introducing a scale hierarchical probabilistic atlas. The algorithm consists of image-space division and a multi-scale weighting scheme. The generated spatial-divided probabilistic atlas efficiently reduces the inter-subject variance in organ shape and position either in global or local regions. Our proposed method was evaluated using 100 abdominal CT volumes with manually traced ground truth data. Experimental results showed that it can segment the liver, spleen, pancreas, and kidneys with Dice similarity indices of $95.1 \%$, $91.4 \%, 69.1 \%$, and $90.1 \%$, respectively.
\end{abstract}

\section{Introduction}

Abdominal organ segmentations from medical images are crucial tasks in medical imaging. Organ region information can be utilized for such purposes as computeraided diagnosis or computer-assisted surgery. Recently, statistical shape models (SSM) [1 5] or probabilistic atlases (PA) 6-10] both of which are statistically computed from multi-atlases, are widely used for abdominal organ segmentation. Both SSMs and PAs are generally registered to the target images as prior knowledge. By incorporating such prior knowledge into the post-processing, organs of interest can be segmented.

In abdominal organ segmentation, traditional SSM-based methods are specialized for a particular organ, such as the liver [1] or the pancreas [2, 4]. Recently, SSM-based methods have also been applied to multi-organ segmentation [3, 5]. 
Although SSM-based methods have high ability for abdominal organ segmentation, it remains difficult to construct an ideal "mean shape" of an organ. Due to large inter-subject differences, the "mean shape" of a special dataset may not exist. Furthermore, since SSMs cannot represent the positional information of an organ, it is difficult to register an SSM to the organ of the segmentation target.

Unlike SSMs, PA is relatively easy to register a PA to an target image. Park et al. first applied PA to abdominal organ segmentation 6. This approach was improved for multi-organ segmentation by Shimizu et al. [7], Oda et al. [8], and Linguraru et al. [9, 10]. Recently, methods based on target-specific PAs [2, 11] has been proposed for abdominal organ segmentation. Instead of generating population-based PAs prior to the segmentation process, target-specific PAs are dynamically generated for unlabeled target images by selecting suitable atlases that are specialized to unlabeled target images based on the atlas-and-target similarities in a global view. Since global similarity does not represent the local difference between the atlases and the target images, important local information may be overlooked in PA generation.

A hierarchical registration method integrated with a weighting scheme improved segmentation accuracy. Wolz et al. [11] proposed a hierarchical atlas registration method that is integrated with a weighting scheme in global, organ, and pixel levels. In this paper, we propose an approach that is more sensitive to inter-subject differences both in the global and local views. By applying hierarchical divisions to image space, we generate spatial-divided PAs that are specific to each unlabeled target image for organ segmentation. Such PAs are generated by assigning a global weight and local weights to each atlas. We use the generated PA to segment the organ areas with a maximum a posterior (MAP) estimation and a graph cut method [12].

\section{Organ Segmentation Based on Spatial-Divided PAs}

\subsection{Overview}

In our method, all atlases are aligned using a spatial normalization method in the pre-processing step. Each unlabeled target image is also normalized by the same process before segmentation. After that, we hierarchically divide the global image space into $N$ sub-spaces:

$$
N=\left(2^{k}\right)^{3} \quad(k=0,1,2, \cdots, M),
$$

where the scale level of $k$ is decided by equally dividing an image into $2^{k}$ parts along the $x-, y-$, and $z$ - axes, respectively. As shown in Fig. 1, number $N$ of sub-spaces increases in the order of $1 \rightarrow 8 \rightarrow 64 \rightarrow 512 \rightarrow \cdots$ with increasing scale-level $k$. On each scale level $k$, atlas-and-target registration is performed based on the MRF non-rigid registration method [13] in each sub-space.

We used a multi-scale weighting scheme to define "global weight" and "local weights" for PA generation. The image similarity between each atlas and target image on the global image space $(N=1)$ is used to define the global weight. 


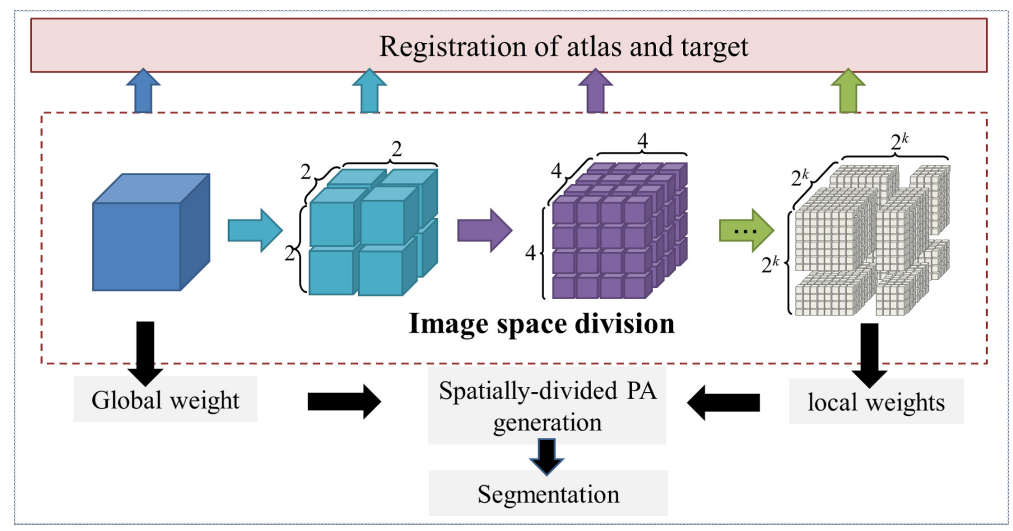

Fig. 1. In our proposed method, the image space is hierarchically divided into subspaces. Atlas-and-target registrations are implemented in each step. Gobal and local weights between each atlas and target image are obtained for $N=1, N=\left(2^{k}\right)^{3}$. These weights are then used to generate PAs for organ segmentation.

The image similarities between each atlas and the target image on the sub-spaces $\left(N=\left(2^{k}\right)^{3}\right)$ are computed to define the local weights. Using the global weight and the local weights, a spatial-divided PA that is specific to the target image is generated. Finally, the generated PA is used to segment the organ areas using a MAP estimation and a graph cut method.

\subsection{Spatial Normalization}

Spatial normalization is achieved by aligning all atlases to a common space based on scaling and translation with $\mathbf{p}^{\prime}=\mathbf{p} \mathbf{T}_{1} \mathbf{S} \mathbf{T}_{2}$, where $\mathbf{p}$ is the coordinate of an voxel in the original atlas space and $\mathbf{p}^{\prime}$ is the transformed coordinate of this voxel. Translation matrices $T_{1}$ and $T_{2}$ and scaling matrix $\mathbf{S}$ are defined as

$$
\mathbf{T}_{1}=\left[\begin{array}{llll}
1 & 0 & 0 & 0 \\
0 & 1 & 0 & 0 \\
0 & 0 & 1 & 0 \\
-C_{x}-C_{y}-B_{z} & 1
\end{array}\right], \mathbf{T}_{2}=\left[\begin{array}{llll}
1 & 0 & 0 & 0 \\
0 & 1 & 0 & 0 \\
0 & 0 & 1 & 0 \\
\overline{C_{x}} & \overline{C_{y}} & \bar{B}_{z} & 1
\end{array}\right], \mathbf{S}=\left[\begin{array}{cccc}
\frac{w}{\bar{w}} & 0 & 0 & 0 \\
0 & \frac{h}{h} & 0 & 0 \\
0 & 0 & \frac{d}{d} & 0 \\
0 & 0 & 0 & 1
\end{array}\right]
$$

All of the parameters in these matrices are automatically computed from the extracted abdomen, the lung area, and the kidney area. Here, the $C_{x}$ and $C_{y}$ are the values of the centroid point of the abdomen in the axial direction $x$ and $y, B_{z}$ is the position of the bottom of the lung area in axial direction, $w \mathrm{~mm}$ and $h \mathrm{~mm}$ are the width and height of the abdomen area, and $d$ is the distance between the bottom of the lung area and the kidneys in the $z$-axis. All the other parameters of $\bar{C}_{x}, \bar{C}_{y}, \bar{B}_{z}, \bar{w}, \bar{h}$ and $\bar{d}$ are the average values statistically computed from multiple atlases. In addition, we interpolate all of the atlases and the target images to isotropic voxels. 


\subsection{PA Generation}

For each voxel $\mathbf{p}$ located in sub-space $U_{j}$, the probability that each voxel $\mathbf{p}$ belongs to organ $l$ is repeatedly calculated by

$$
\Lambda_{\mathbf{p} \in U_{j}}(l)=\sum_{i} \omega_{i}^{g} \omega_{i j}^{s} \delta\left(L_{\mathbf{p}}^{i}, l\right) / \sum_{i} \omega_{i}^{g} \omega_{i j}^{s},
$$

where

$$
\delta\left(l, l^{\prime}\right)=\left\{\begin{array}{l}
1 \text { if } l=l^{\prime} \\
0 \text { otherwise. }
\end{array}\right.
$$

Here, $i$ represents the index of the atlases, $j$ represents the index of the subspaces, $l$ represents the label of each organ, and $L_{\mathbf{p}}^{i}$ is the label of voxel $\mathbf{p}$ in the manual segmentation of atlas $i . \omega_{i}^{g}$ is the global weight and $\omega_{i j}^{s}$ is the local weight in sub-space $U_{j}$ between atlas $\mathcal{A}_{i}$ and target image $\mathcal{I}$. $\omega_{i}^{g}$ and $\omega_{i j}^{s}$ are explained below.

Global Weight Calculation. For a given target image, the image similarity between each atlas $\mathcal{A}_{i}$ and target image $\mathcal{I}$ on the global image space $(N=1)$ is calculated. Here, normalized cross correlation (NCC) is used for the similarity evaluation:

$$
\operatorname{NCC}\left(\mathcal{A}_{i}, \mathcal{I}\right)=\operatorname{Cov}\left(\mathcal{A}_{i}, \mathcal{I}\right) /\left(\sqrt{\operatorname{Var}\left(\mathcal{A}_{i}\right)} \sqrt{\operatorname{Var}(\mathcal{I})}\right),
$$

where $\operatorname{Cov}\left(\mathcal{A}_{i}, \mathcal{I}\right)$ is the covariance of atlas $\mathcal{A}_{i}$ and target image $\mathcal{I}$, $\operatorname{Var}\left(\mathcal{A}_{i}\right)$ and $\operatorname{Var}(\mathcal{I})$ are the variances of atlas $\mathcal{A}_{i}$ and target image $\mathcal{I}$. The global weight of atlas $\mathcal{A}_{i}$ is then defined by

$$
\omega_{i}^{g}\left(\mathcal{A}_{i}\right)=1-\operatorname{NCC}\left(\mathcal{A}_{i}, \mathcal{I}\right) .
$$

Local Weight Calculation. On each divided sub-space $U_{j}(j=1, \cdots, N)$, we calculate the similarities between atlas $\mathcal{A}_{i}$ and target image $\mathcal{I}$ based on the sum of the squared intensity differences (SSD). The SSD between atlas $\mathcal{A}_{i}$ and target image $\mathcal{I}$ in each sub-space $U_{j}$ is then normalized into the range of $[0,1]$ to define the local weight as

$$
\omega_{i j}^{s}\left(U_{j}\right)=1-\operatorname{SSD}_{U_{j}}\left(\mathcal{A}_{i}, \mathcal{I}\right) .
$$

\subsection{Organ Segmentation}

We obtain a coarse segmentation result using a MAP estimation that is defined by

$$
C_{\mathbf{p}}=\arg \max _{l} \operatorname{Pr}_{\mathbf{p}}\left(I_{\mathbf{p}} \mid l\right) \operatorname{Pr}_{\mathbf{p}}(l),
$$

where $C$ is the coarse segmentation result, $\mathbf{p}$ is voxel in the target image, and $l$ represent the label of each organ. $\operatorname{Pr}_{\mathbf{p}}(l)$ is the prior probability of organ $l$, which is given by the generated PA. $\operatorname{Pr}_{\mathbf{p}}\left(I_{\mathbf{p}} \mid l\right)$ is the intensity distribution of 
Table 1. Mean and SD of dice similarity index (DSI) and average surface distance (ASD) of $100 \mathrm{CT}$ volumes. Results of different scale level of sub-space $k$ are shown.

\begin{tabular}{c|c|c|c|c||c|c|c|c}
\hline & \multicolumn{3}{|c||}{ Dice similarity index (\%) } & \multicolumn{3}{c}{ Average surface distance (mm) } \\
\hline & Liver & Spleen & Pancreas & Kidneys & Liver & Spleen & Pancreas & Kidneys \\
\hline$N=1$ & $95.1 \pm 1.0$ & $90.6 \pm 5.8$ & $67.4 \pm 15.5$ & $89.6 \pm 5.4$ & $1.21 \pm 0.2$ & $0.92 \pm 0.5$ & $1.98 \pm 0.6$ & $1.30 \pm 0.4$ \\
\hline$N=8$ & $95.1 \pm 1.0$ & $90.9 \pm 5.9$ & $67.8 \pm 15.5$ & $89.6 \pm 5.6$ & $1.21 \pm 0.2$ & $0.91 \pm 0.5$ & $1.97 \pm 0.7$ & $1.30 \pm 0.4$ \\
\hline$N=64$ & $95.1 \pm 1.0$ & $91.4 \pm 5.7$ & $69.1 \pm 15.3$ & $90.1 \pm 5.0$ & $1.20 \pm 0.2$ & $0.89 \pm 0.4$ & $1.88 \pm 0.6$ & $1.26 \pm 0.4$ \\
\hline$N=512$ & $94.6 \pm 1.5$ & $89.4 \pm 8.3$ & $65.7 \pm 19.5$ & $88.1 \pm 8.5$ & $1.25 \pm 0.3$ & $1.0 \pm 0.5$ & $1.89 \pm 0.7$ & $1.35 \pm 0.5$ \\
\hline
\end{tabular}

each organ $l$, which is approximated by Gaussian distribution $\operatorname{Pr}_{\mathbf{p}}\left(I_{\mathbf{p}} \mid \mu_{l}, \sigma_{l}^{2}\right) . \mu_{l}$ and $\sigma_{l}^{2}$ are estimated by an EM algorithm.

We assign organ label $l$ to each voxel p based on Eq. (8) to obtain the coarse segmentation result, which is then refined by following the graph cut method shown in 8, 12 .

\section{$3 \quad$ Experiments and Results}

We evaluated our proposed method using a diverse dataset of 100 portal-phased abdominal CT volumes. The acquisition parameters of the CT volumes are 512512 pixels, 263-538 slices, and $0.546-0.820 \mathrm{~mm}$ of pixel spacing. All of the CT volumes were acquired in the period from 2004 to 2009. The ages of the patients in the CT volumes ranges from 26 to 83. Manual segmentations were generated by one of three trained raters using a semi-automatic method based on region growing and graph cut method. After the semi-automated segmentation, a slice-by-slice manual correction process is performed by an expert rater for all the CT images.

Four organs, the liver, spleen, pancreas, and the kidneys were selected as segmentation objects. Each atlas was in turn segmented using a leave-one-out cross validation method. We evaluated the segmentation performance using the Jaccard index (JI), the Dice similarity index (DSI), and the average surface distance (ASD) defined in 1]. For each case of sub-space number $N=$ $1,8,64,512(k=0,1,2,3)$, we evaluated the segmentation results (Table 11). We also compared the segmentation results to state-of-the-art methods of abdominal organ segmentation (Table 2). Fig. 2 gives examples of the segmentation results. Fig. 3 shows the changes of the generated PAs with a different number of sub-spaces.

\section{Discussion}

We presented a spatially-divided PA generation method based on hierarchical sub-space divisions. We applied spatially-divided PA to abdominal organs segmentation to deal with the local inter-subject differences in organ shape and position. Table 1 shows that the segmentation accuracy of the spleen, pancreas, 
Table 2. Comparison of segmentation performance with SSM- and PA-based methods. Results are shown using average JI and ASD. (Middle group: Single organ segmentation, lower group: Multi-organ segmentation).

\begin{tabular}{c|c||c|c|c|c||c|c|c|c}
\hline \multicolumn{1}{c||}{} & \multicolumn{4}{c||}{ Jaccard index (\%) } & \multicolumn{3}{c}{ Average surface distance $(\mathrm{mm})$} \\
\hline Method & Cases & Liver & Spleen & Pancreas & Kidneys & Liver & Spleen & Pancreas & Kidneys \\
\hline Proposed & 100 & 90.6 & 84.5 & 54.6 & 82.3 & 1.20 & 0.89 & 1.88 & 1.26 \\
\hline \hline Okada [3] & 28 & 88.8 & - & - & - & 1.46 & - & - & - \\
Heimann [1] & 35 & 92.3 & - & - & - & 1.40 & - & - & - \\
Shimizu [2] & 20 & - & - & 57.9 & - & - & - & - & - \\
Erdt [4] & 40 & - & - & 61.2 & - & - & - & 1.70 & - \\
\hline \hline Chen [5] & 20 & - & - & - & - & 0.81 & 0.75 & - & 0.77 \\
Shimizu [7] & 10 & 89.0 & 83.5 & 35.0 & 82.5 & - & - & - & - \\
Oda [8] & 100 & 89.0 & 74.5 & 42.1 & 80.8 & - & - & - & - \\
Linguraru 9] & 20 & 92.7 & 91.0 & - & - & 1.2 & 0.7 & - & - \\
Linguraru [10] & 40 & - & - & - & - & 3.0 & 2.1 & - & 1.8 \\
Wolz [1] & 100 & 89.5 & 84.6 & 49.6 & 88.1 & - & - & - & - \\
\hline
\end{tabular}

and the kidneys are statistically improved (paired t-test, $p<0.01$ ) when subspace number $N$ increased from 1 to 64 . By adding a local weight to each subregion, the local variations among patients in organ appearance were significantly reduced by our proposed method. This can be observed in Fig. 3 . The PA of $N=64$ matches the organs in the target image more closely, leading to high segmentation accuracy. On the other hand, segmentation accuracy decreased for $N=512$. Since the size of the sub-space becomes smaller for $N=512$, the texture information and the relationship among neighborhood voxels failed. In this case, the atlas-and-target registrations in the small local areas cannot guar-
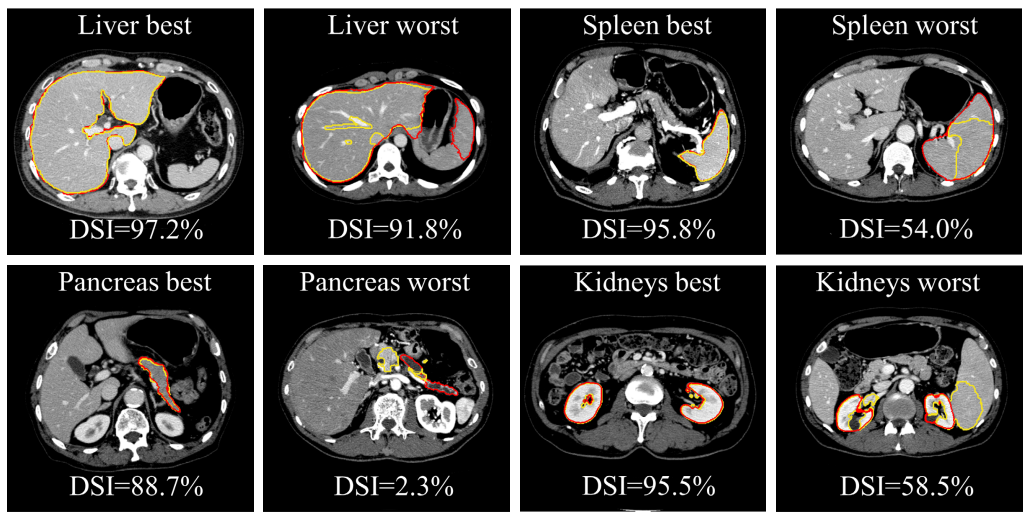

Fig. 2. Worst and best segmentation results for liver, spleen, pancreas, and kidneys. Segmentation results are outlined in yellow lines and manual segmentation result is shown in red. Dice similarity indices are given. 

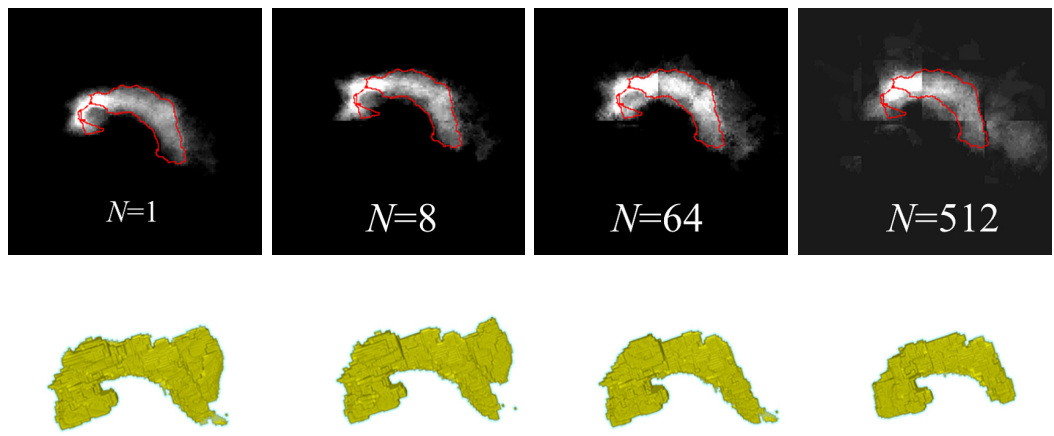

DSI $=61.6 \%$

DSI $=65.8 \%$

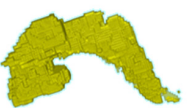

$\mathrm{DSI}=75.4 \%$

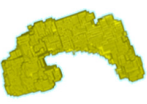

$\mathrm{DSI}=70.3 \%$

Fig. 3. Examples of generated PAs in different sub-space numbers of $N=1,8,64,512$. First row gives axial slices of generated PAs. Second row shows correspondence segmentation results based on the above PAs.

antee a fine global appearance of generated PAs. Experiments are necessary to determine the most suitable $N$ in the future.

Table 2 shows that our proposed method achieves a relatively favorable performance compared to the state-of-the-art methods. Especially on the pancreas, which is the most challenging structure, the segmentation accuracy has been greatly improved compared to the previous PA-based methods [7-10]. Although the accuracy of the pancreas in our experiment is not as high as in the SSMbased pancreas-specific method [2, 4, the results remain acceptable that were obtained from a large and diverse atlas database. Note that previous work [2,4] only tested small number of cases.

The run-time of the PA generation took more than two hours for each target image. Since $99 \mathrm{CT}$ volumes must be registered to the target image both in the global space and the sub-spaces, a significant number of registrations are required. Increasing the speed of the proposed approach is expected by selecting a suitable sub-set of atlases based on a fast similarity comparison.

\section{Conclusion}

In this paper, we proposed a multi-organ segmentation method using locally generated target-specific PAs. We evaluated the segmentation performance of our proposed method using $100 \mathrm{CT}$ volumes and obtained 95.1\%, 91.4\%, 69.1\%, and $90.1 \%$ of the Dice similarity indices for the liver, spleen, pancreas, and the kidneys, respectively. Future work includes the addition of the shape information for the pancreas segmentation, and the reduction of the computation time by developing of fast registration and similarity comparison methods.

Acknowledgments. The authors thank our colleagues for suggestions and advice. Parts of this research were supported by RS-JSPS Research Cooperative 
Program, MEXT/JSPS KAKENHI, and the Kayamori Foundation of Informational Science Advancement.

\section{References}

1. Heimann, T., et al.: Comparison and evaluation of methods for liver segmentation from CT datasets. IEEE TMI 28(8), 1251-1265 (2009)

2. Shimizu, A., Kimoto, T., Kobatake, H., Nawano, S., Shinozaki, K.: Automated pancreas segmentation from three-dimensional contrast-enhanced computed tomography. Int. J. CARS 5, 85-98 (2010)

3. Okada, T., Yokota, K., Hori, M., Nakamoto, M., Nakamura, H., Sato, Y.: Construction of Hierarchical Multi-Organ Statistical Atlases and Their Application to Multi-Organ Segmentation from CT Images. In: Metaxas, D., Axel, L., Fichtinger, G., Székely, G. (eds.) MICCAI 2008, Part I. LNCS, vol. 5241, pp. 502-509. Springer, Heidelberg (2008)

4. Erdt, M., Kirschner, M., Drechsler, K., Wesarg, S., Hammon, M., Cavallaro, A.: Automatic pancreas segmentation in contrast enhanced CT data using learned spatial anatomy and texture descriptors. In: IEEE ISBI 2011, pp. 2076-2082 (2011)

5. Chen, X., Udupa, J.K., Bagci, U., Zhuge, Y., Yao, J.: Medical image segmentation by combining graph cuts and oriented active appearance models. IEEE TMI 21(4), 2035-2046 (2012)

6. Park, H., Bland, P., Meyer, C.: Construction of an abdominal probabilistic atlas and its application in segmentation. IEEE TMI 22(4), 483-492 (2003)

7. Shimizu, A., Ohno, R., Ikegami, T., Kobatake, H., Nawano, S., Smutek, D.: Segmentation of multiple organs in non-contrast 3D abdominal CT images. Int. J. CARS, 135-142 (2007)

8. Oda, M., Nakaoka, T., Kitasaka, T., Furukawa, K., Misawa, K., Fujiwara, M., Mori, K.: Organ segmentation from 3D abdominal CT images based on atlas selection and graph cut. In: Yoshida, H., Sakas, G., Linguraru, M.G. (eds.) Abdominal Imaging 2011. LNCS, vol. 7029, pp. 181-188. Springer, Heidelberg (2012)

9. Linguraru, M.G., Sandberg, J.A., Li, Z., Shah, F., Summers, R.M.: Atlas-based automated segmentation of spleen and liver using adaptive enhancement estimation. Medical Physics 37(2), 771-783 (2010)

10. Linguraru, M.G., Pura, J.A., Pamulapati, V., Summers, R.M.: Statistical 4D graphs for multi-organ abdominal segmentation from multiphase CT. Medical Image Analysis 16(4), 904-914 (2012)

11. Wolz, R., Chu, C., Misawa, K., Mori, K., Rueckert, D.: Multi-organ Abdominal CT Segmentation Using Hierarchically Weighted Subject-Specific Atlases. In: Ayache, N., Delingette, H., Golland, P., Mori, K. (eds.) MICCAI 2012, Part I. LNCS, vol. 7510, pp. 10-17. Springer, Heidelberg (2012)

12. Boykov, Y., Veksler, O., Zabih, R.: Fast approximate energy minimization via graph cuts. IEEE PAMI 23(11), 1222-1239 (2001)

13. Glocker, B., Komodakis, N., Tziritas, G., Navab, N., Paragios, N.: Dense image registration through MRFs and efficient linear programming. Medical Image Analysis 12(6), 731-741 (2008) 